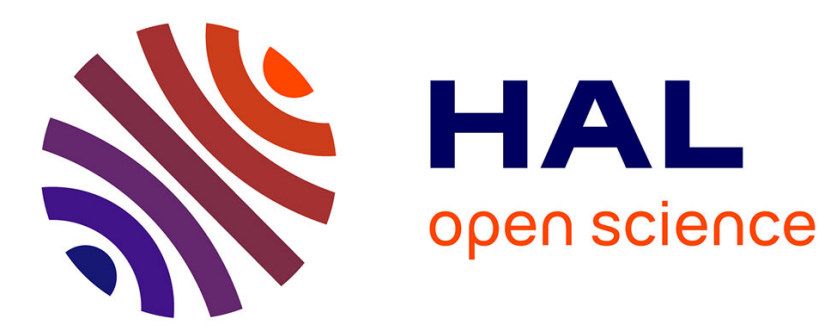

\title{
Influence of the player on the dynamics of the electric guitar
}

Jean-Loic Le Carrou, Arthur Paté, Baptiste Chomette

\section{To cite this version:}

Jean-Loic Le Carrou, Arthur Paté, Baptiste Chomette. Influence of the player on the dynamics of the electric guitar. Journal of the Acoustical Society of America, 2019, 146 (5), pp.3123-3130. 10.1121/1.5130894. hal-02495624

\section{HAL Id: hal-02495624 \\ https://hal.sorbonne-universite.fr/hal-02495624}

Submitted on 2 Mar 2020

HAL is a multi-disciplinary open access archive for the deposit and dissemination of scientific research documents, whether they are published or not. The documents may come from teaching and research institutions in France or abroad, or from public or private research centers.
L'archive ouverte pluridisciplinaire HAL, est destinée au dépôt et à la diffusion de documents scientifiques de niveau recherche, publiés ou non, émanant des établissements d'enseignement et de recherche français ou étrangers, des laboratoires publics ou privés. 
Influence of the player on the dynamics of the electric guitar

Jean-Loic Le Carrou, ${ }^{1, a)}$ Arthur Paté, ${ }^{2}$ and Baptiste Chomette ${ }^{1}$

${ }^{1}$ Sorbonne Université, CNRS, Institut Jean Le Rond d'Alembert, Paris,

France

${ }^{2}$ Univ Lille, CNRS, École Centrale, ISEN, Univ Valenciennes, IEMN, UMR 8520,

Lille , France 
The sound of the electric guitar is strongly dependent on the string vibration. Where a mode of the structure coincides with a mode of the fretted string, coupling between the string and structure occurs at that "deadspot." The coupling significantly lowers decay time, leading to the name (Paté et al., 2014). But how the guitarist affects the dynamic behavior of the structure by grasping the neck, holding the instrument with the strap, or laying the instrument on his/her thigh remains to be investigated. This is the aim of the paper. Two methods are proposed to identify the modal parameters of the electric guitar structure, either by a classical modal analysis in simulated playing configuration, or by an operational modal analysis (OMA) in real playing configuration. For this latter method, modal parameters are identified from dynamic measurements performed when each string is plucked. Both methods are compared and allow us to quantify the modal frequency modification and the added modal damping, that depend on the player's body-part in contact with the structure and on the modal shape considered. Consequences of these modal parameters on the modeled sound show that the player can increase the decay time close to a deadspot.

a)jean-loic.le_carrou@sorbonne-universite.fr 


\section{INTRODUCTION}

The sound of the electric guitar comes from the conversion of the mechanical vibration of the string into an electric signal. Consequently, the sound does not seem to depend on the mechanical properties of the guitar body. However, in the general case of stringed musical instruments, the modal parameters of the instrument body may affect the vibration of the string. While there are few studies on the electric guitar, much research on violin and acoustic guitar dealt with coupling phenomena in the case of the violin or the guitar. For example, Gough (Gough, 1980, 1981) studied the coupling between the string and the body using an analytical model in the case of the wolf-note phenomenon that appears in the violin. Woodhouse (Woodhouse, 2004a’b) proposed several sound synthesis methods and underlined the connection between the modal parameters of the acoustic guitar body and the sound of the instrument. More recently, Benacchio et al. (Benacchio et al., 2016) experimentally demonstrated the importance of the modal parameters of the guitar body in the sound of the instrument using active modal control. In the case of the electric guitar, body-coupling may also, in some cases, affect the string vibration, mostly when there is a frequency coincidence between string and body modes. Paté et al. (Paté et al., 2014) showed that the decay time of solid body electric guitar tones is due to the combined action of string's intrinsic damping and coupling-induced damping. It was notably shown that for the electric guitar, this coupling mainly occurs at the neck (Fleischer and Zwicker, 1999; Paté et al., 2014). However, in the classic way of playing the electric guitar, ergonomic studies (Marmaras and Zarboutis, 1997) showed that the left-hand palm holds the neck and 
the left-hand finger presses the string against the fingerboard. Thus, the left hand may have consequences on the neck vibration. Similarly, the player's body (e.g. stomach, thigh) is in contact with the body of the instrument and may also modify the instrument's vibration. In order to measure these effects, previous studies used simulated playing configuration while the instrument was excited by a classical system. For the electric guitar, the experimenter held the neck, on which the shaker is fixed, puts the instrument on his knees, and the left hand grasped the neck (Fleischer, 2005; Fleischer and Zwicker, 1998, 1999). For the violin, excited by an impact hammer, the experimenter held the instrument between his chin and his shoulder in a "usual manner" (Marshall, 1986). Results of these first studies clearly show that the player increases the damping of the structure depending on the mode. But this experimental methodology is quite far from a real playing posture. However, musical instruments contain their own excitation system that can be used to identify their modal basis with Operational Modal Analysis method (OMA), as performed recently on a concert harp (Chomette and Le Carrou, 2015).

The aim of this paper is to identify the modal basis of the electric guitar when it is played in order to quantify the influence of the player on the dynamic behavior of the electric guitar structure. The operational modal analysis is presented in Section II. The experimental method is proposed in Section III. Results are shown in Section IV and a discussion highlighting the influence of the player both on the electric guitar vibration and on the sound of the instrument is given in Section V. 


\section{OPERATIONAL MODAL ANALYSIS IN TIME DOMAIN}

The aim of the Operational Modal Analysis (OMA) is to identify modal parameters using only measured data without knowing the excitation. In the case of an unknown impulse response, OMA methods can use the Linear Square Complex Exponential (LSCE) algorithm introduced by Brown et al. (Brown et al., 1979). In this method, the time response of a structure $h_{i j}(k \Delta t)$ at the $k t h$ time sample $\Delta t$ located at point $i$ due to an impulse located at point $j$ can be expressed as the summation of $N$ decaying sinusoids whose frequency and damping ratio are associated to the $r t h$ structural mode

$$
h_{i j}(k \Delta t)=\sum_{r=1}^{N} \frac{\phi_{r i} A_{r j}}{m_{r} \omega_{r}^{d}} e^{-\xi_{r} \omega_{r}^{n} k \Delta t} \sin \left(\omega_{r}^{d} k \Delta t+\theta_{r}\right)
$$

where $\omega_{r}^{n}$ and $\omega_{r}^{d}=\omega_{r}^{n} \sqrt{1-\xi_{r}^{2}}$ are the non-damped and damped frequency respectively. $\xi_{r}$ is the damping ratio. $\phi_{r i}$ is the $i t h$ component of the $r t h$ mode. $A_{r j}, m_{r}^{d}$ and $\theta_{r}$ are a constant associated to the $j t h$ response signal, the $r t h$ modal mass and the phase angle of the $r$ th modal response respectively. The impulse response can also be written numbering all complex modes and poles including conjugates from $r=1$ to $r=2 \mathrm{~N}$

$$
h_{i j}(k \Delta t)=\sum_{r=1}^{2 N} C_{r i j} e^{s_{r} k \Delta t}
$$

where $C_{r i j}$ is the complex amplitude of the $r t h$ mode for the $i t h$ input and the $j t h$ output. The poles $s_{r}=\omega_{r}^{n} \xi_{r} \pm j \omega_{r}^{d}$ associated to the modes of the structure appear in complex conjugate form. Consequently the complex exponentials $V_{r}=e^{s_{r} \Delta t}$ are the roots of the polynomial Prony's equation of order $2 N$

$$
\beta_{0}+\beta_{1} V_{r}^{1}+\cdots+\beta_{2 N-1} V_{r}^{2 N-1}+V_{r}^{2 N}=0,
$$


with $\beta_{2 N}=1$. By multiplying equation 2 by $\beta_{k}$ and sum over $k=0 \cdots 2 N$, equation 3 gives

$$
\sum_{k=0}^{2 N} \beta_{k} h_{i j}(k \Delta t)=0
$$

By writting $2 N$ times equation 4 starting at successive sample times, the coefficients $\beta_{k}$ are the roots of a linear system. In practice, the system is overdetermined to increase the robustness of the method and is thus solved using the least square method. The poles are finally obtained using

$$
s_{r}=\frac{1}{\Delta t}\left(\left|V_{r}\right| \pm j \arg \left(V_{r}\right)\right) \text {, }
$$

where arg denotes the argument of the complex poles. In practice, the stable poles are automatically extracted using a stabilization chart (Chomette and Mamou-Mani, 2018). This diagram is based on several runs of the pole identification process by using models of increasing order $N$. Physical poles always appear around the same frequency whereas mathematical poles tend to span the whole frequency range. The typical stabilization criteria are chosen as equal to $1 \%$ for the frequency and $5 \%$ for the damping. Poles are considered to be stable if their identified frequency and damping do not exceed theses values between two successive runs at order $n$ and $n+1$.

In the case of a white noise excitation, OMA methods can be based on the Natural Excitation Technique (NExT) introduced by James et al (James et al., 1995). If damping is small, the main assumption of the NExT method is that the correlation function between two sensors located at points $i$ and $j$ can be written as the impulse response function located at point $i$ due to an impulse at point $j$. Using the correlation function, the method is then similar to the LSCE method. In the case of string instruments, Chomette and Le Carrou 


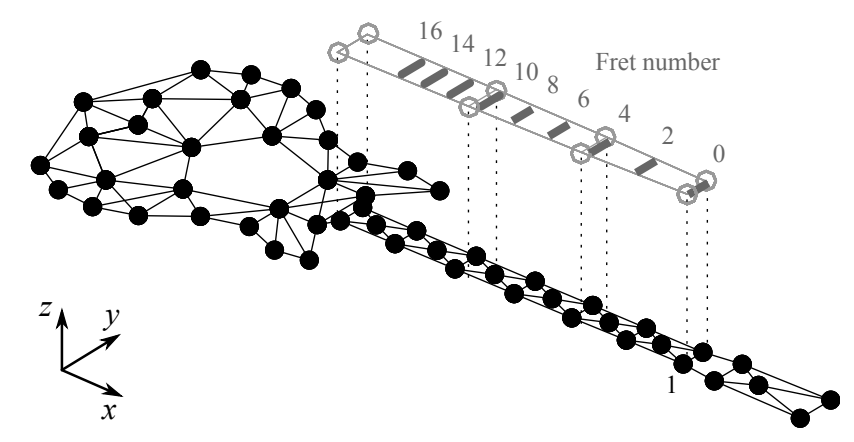

FIG. 1. Two meshes used in the study: 54-point on the whole electric guitar (black) on which point label 1 is shown and 8-point on the neck (gray) on which Frets 0 to 16 are shown.

(Chomette and Le Carrou, 2015) have shown that the NExT-LSCE method can be applied successfully for a plucked string instrument: the concert harp. Indeed, the excitation induced by a string on the instrument can be considered as a sum of damped harmonic components. If the harmonic frequencies of the string are well separated from the eigenfrequencies of the structure, modal parameters can be easily identified. If the harmonic frequencies of the string are close to the structural mode frequencies, modified methods must be used (Marshall, 1986; Mohanty and Rixen, 2004).

\section{EXPERIMENTAL METHOD}

In order to identify the modal parameters of the electric guitar, two methods are performed: a classical modal analysis and an operational modal analysis (OMA). For the former, one or a few accelerometers are glued on the guitar while an impact hammer successively hits different points of the experimental mesh. The classical analysis is performed on the 54-point and the 8-point meshes shown in Figure 1, whereas for the OMA only the 8-point 

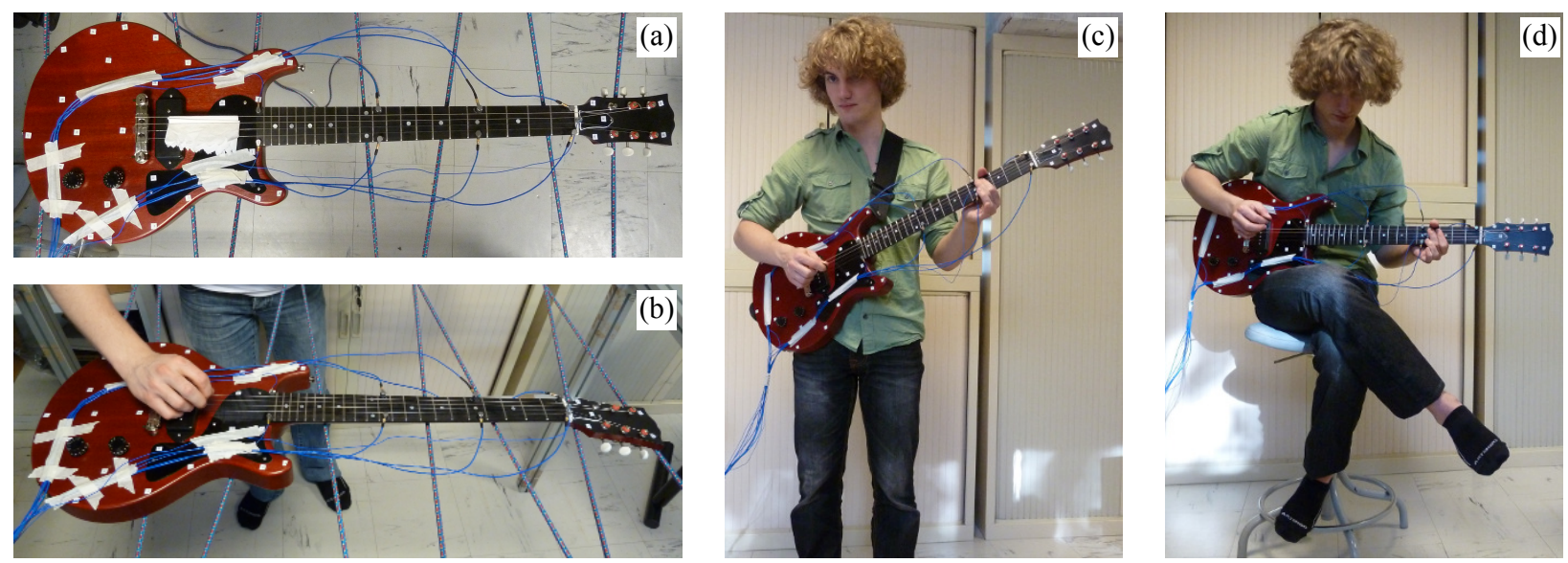

FIG. 2. Experimental configurations for classical modal analysis when the electric guitar is free to vibrate with damped strings (a) and for operational modal analysis in free-free configuration (b) and when the player is standing (c) or sitting (d).

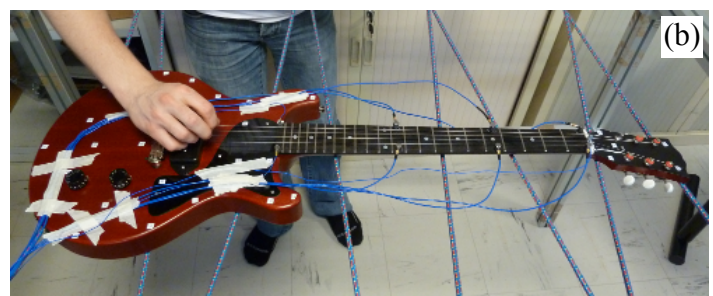

mesh is used. For the classical modal identification, the Least Square Complex Frequency (LCSF) algorithm (Guillaume et al., 2003) (implemented in Modan software) is used. For the OMA, the 8-point mesh is composed of eight accelerometers (PCB M352C65) glued on the neck and another one (PCB 352B10) is moved on the symmetrical axis close to the played fret as shown in Figure 2-(a). This latter accelerometer provides the reference signal for the OMA identification. Note that the location and the size of the eight accelerometers do not allow the first and the sixth string to be mounted on the guitar. Throughout this study, 1d-accelerometers were used, so that only out-of-plane (vertical, i.e. perpendicular to the fingerboard plane) accelerations are measured and shown in this direction only in the following.

In order to quantify the dynamical modification of the instrument when playing, three configurations are tested: with sitting or standing player (i.e. two usual playing configu- 
rations) and with nobody touching the instrument. For the first two configurations, the right-handed player holds the electric guitar with the strap or lays it on his right thigh. For both configurations, the left hand holds the neck and a finger presses the string on the fret to set the vibrating length of the string, as shown in Figures 2-(c) and 2-(d). The last configuration is used as a reference by laying the electric guitar on elastic straps supported by a frame as to simulate free-free boundary conditions (Paté, 2014), as shown in Figure 2-(b).

For the OMA, the player is asked to play several notes along 4 strings $\left(\mathrm{A}_{2}, \mathrm{D}_{3}, \mathrm{G}_{3}\right.$ and $\mathrm{B}_{3^{-}}$ string). The left-hand middle finger presses the string against the fingerboard successively at frets 2 to 16 every two frets. At the nut (denoted F0), the left hand does not hold the neck. The other strings are blocked with the other fingers of the left-hand (this is a common practice for guitar players). All fundamental frequencies of each note played are gathered in Table I.

\section{RESULTS}

\section{A. Classical modal analysis}

\section{Free-free configuration}

A classical modal analysis of the complete electric guitar was performed by using an LSCF method as previously explained. Until $500 \mathrm{~Hz}, 6$ modes are identified. Their modal shapes are displayed in Figure 3. First, two kinds of mode are present: global (1) and local modes (2 to 6) with only neck displacement. Second, among these modes, two are perfect bending 


\begin{tabular}{|c|c|c|c|c|}
\hline Fret & String 5 & String 4 & String 3 & String 2 \\
\hline 0 & $110.00\left(\mathrm{~A}_{2}\right)$ & $146.82\left(\mathrm{D}_{3}\right)$ & $196.00\left(\mathrm{G}_{3}\right)$ & $246.94\left(\mathrm{~B}_{3}\right)$ \\
\hline 2 & $123.47\left(\mathrm{~B}_{2}\right)$ & $164.81\left(\mathrm{E}_{3}\right)$ & $220.00\left(\mathrm{~A}_{3}\right)$ & $277.18\left(\mathrm{C} \#_{4}\right)$ \\
\hline 4 & $138.59\left(\mathrm{C} \#_{3}\right)$ & $184.99\left(\mathrm{~F} \#_{3}\right)$ & $246.94\left(\mathrm{~B}_{3}\right)$ & $311.12\left(\mathrm{D} \#_{4}\right)$ \\
\hline 6 & $155.56\left(\mathrm{D} \#_{3}\right)$ & $207.65(\mathrm{G} \# 3)$ & $277.19\left(\mathrm{C} \#_{4}\right)$ & $349.23\left(\mathrm{~F}_{4}\right)$ \\
\hline 8 & $174.61\left(\mathrm{~F}_{3}\right)$ & $233.08\left(\mathrm{A \#}{ }_{3}\right)$ & $311.13\left(\mathrm{D} \#_{4}\right)$ & $391.99\left(\mathrm{G}_{4}\right)$ \\
\hline 10 & $196.00\left(\mathrm{G}_{3}\right)$ & $261.62\left(\mathrm{C}_{4}\right)$ & $349.23\left(\mathrm{~F}_{4}\right)$ & $440.00\left(\mathrm{~A}_{4}\right)$ \\
\hline 12 & $220.00\left(\mathrm{~A}_{3}\right)$ & $293.66\left(\mathrm{D}_{4}\right)$ & $392.00\left(\mathrm{G}_{4}\right)$ & $493.88\left(\mathrm{~B}_{4}\right)$ \\
\hline 14 & $246.94\left(\mathrm{~B}_{3}\right)$ & $329.22\left(\mathrm{E}_{4}\right)$ & $440.01\left(\mathrm{~A}_{4}\right)$ & $554.36\left(\mathrm{C} \#_{5}\right)$ \\
\hline 16 & $277.18\left(\mathrm{C} \#_{4}\right)$ & $369.99\left(\mathrm{~F} \#_{4}\right)$ & $493.89\left(\mathrm{~B}_{4}\right)$ & $622.25\left(\mathrm{D} \#_{5}\right)$ \\
\hline
\end{tabular}

TABLE I. Fundamental frequency in $\mathrm{Hz}$ and name of played note for each string and each fret.

modes (1 and 6$)$, two are perfect torsional modes (2 and 5) and two are a combination of bending and torsion which are modes 3 and 4 .

\section{Simulated playing configuration}

A first approach to quantify the player's impact on modal parameters of the electric guitar is to carry out a classical modal analysis on an electric guitar. The guitarist then mimics a playing situation on an instrumented guitar with accelerometers glued on the fingerboard whereas an impact is provided by the hammer close to the nut (on point 1, see Figure 1). 


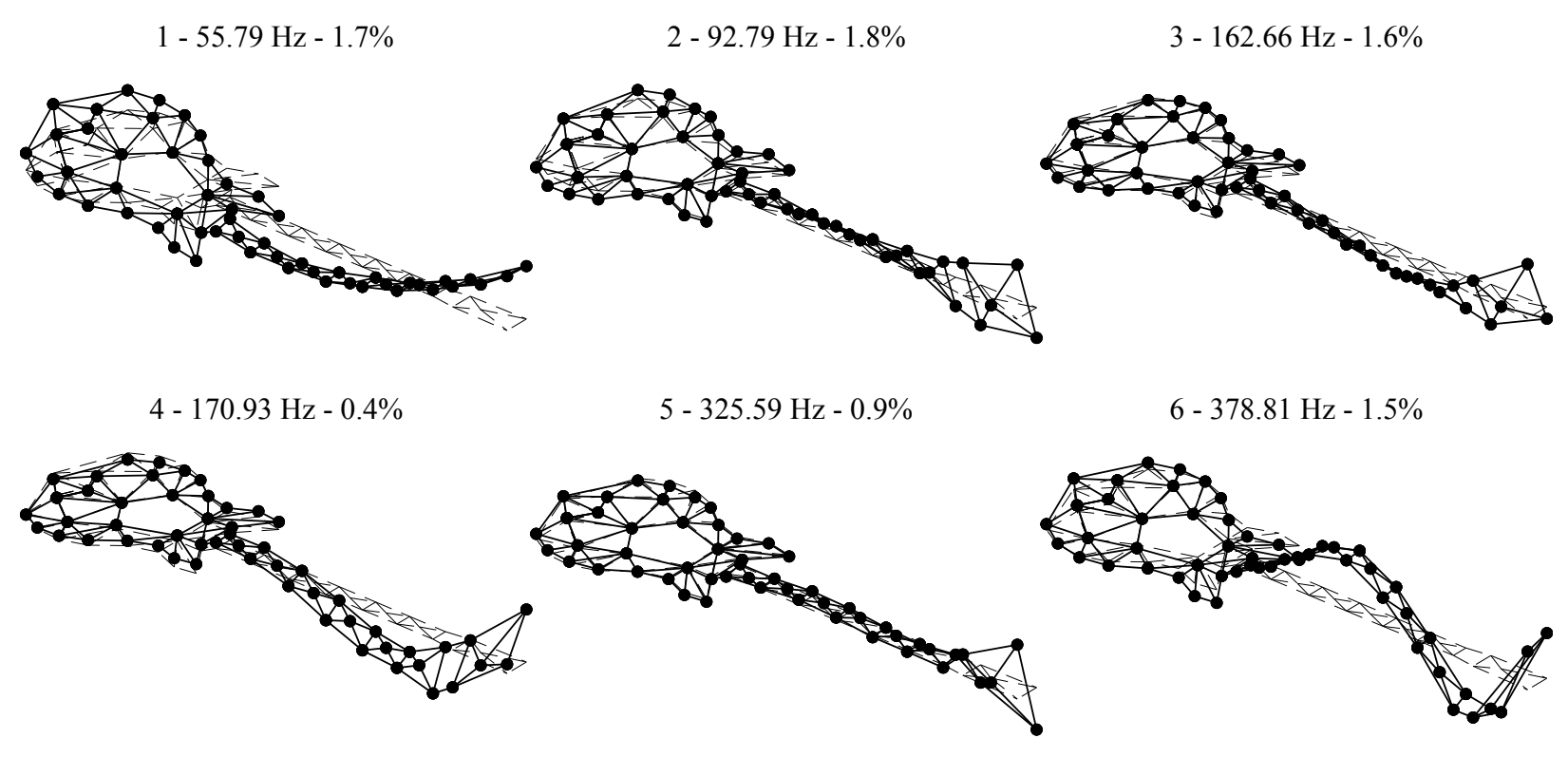

FIG. 3. Modal frequencies, modal damping and modal shapes of the electric guitar in free-free configuration. Dashed lines represent the mesh at rest position, black dots connected by solid lines represent the deformation corresponding to the modal shape.

Results are synthesized in Figure 4 showing each co-localized FRF measured for each lefthand position. In addition, the FRF in free-free condition is also plotted highlighting modes 4 and 6 to be particularly present in the instrument's response. Modal parameters for modes 4 and 6 are gathered in Figure 7.

Results clearly show that holding the electric guitar or letting it lie globally affects the damping of the electric guitar in a similar way for the two player positions. In details, this modification depends on the left-hand position along the neck. Mode 4 is more affected when the guitarist's hand is close to the neck head than mode 6, for instance. The closer the left-hand to the anti-node of the mode, the higher is the damping. Between the two configurations, subtle differences can be seen in terms of damping, especially for mode 4 . 
(a)

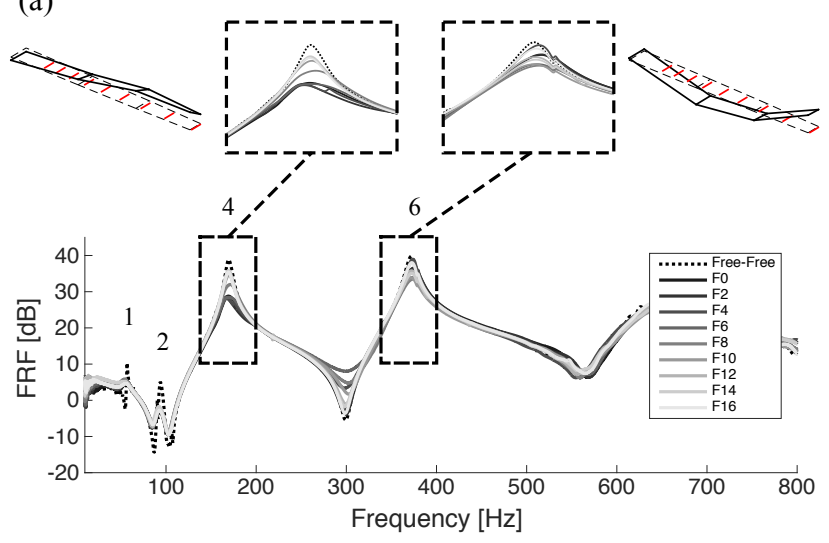

(b)
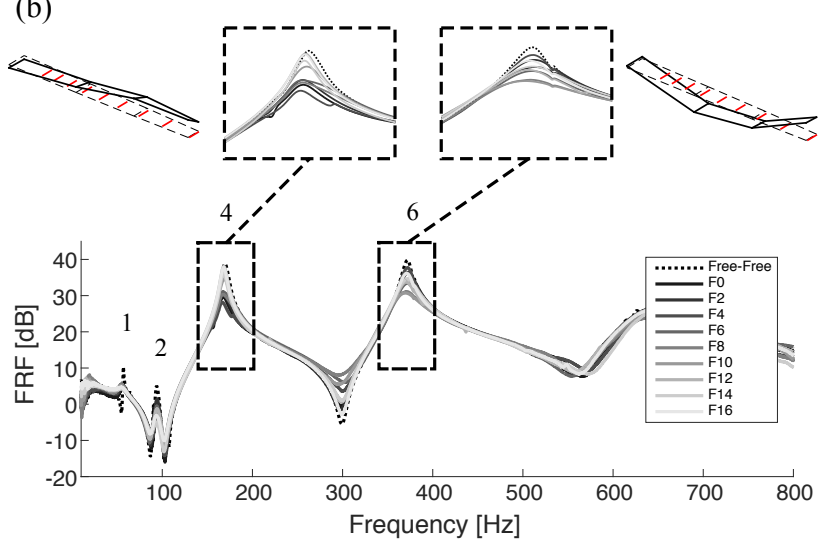

FIG. 4. Co-localized FRF measured at point 1 of the mesh (see Fig. 1) for sitting (a) and standing configurations (b). Numbers refer to mode numbers in Figure 3. FRFs are magnified around the frequency of modes 4 and 6 (corresponding modal shapes are also plotted).

Modes 1 and 2, whose displacement amplitude at point 1 is already low, are also affected by the left hand position along the neck, adding some damping and lowering the FRF amplitudes even more. Note that modes 3 and 5 are not visible in the co-localized FRF, as for these modes the displacement amplitude at point 1 is much lower than for the other modes, as shown in Figure 3. 

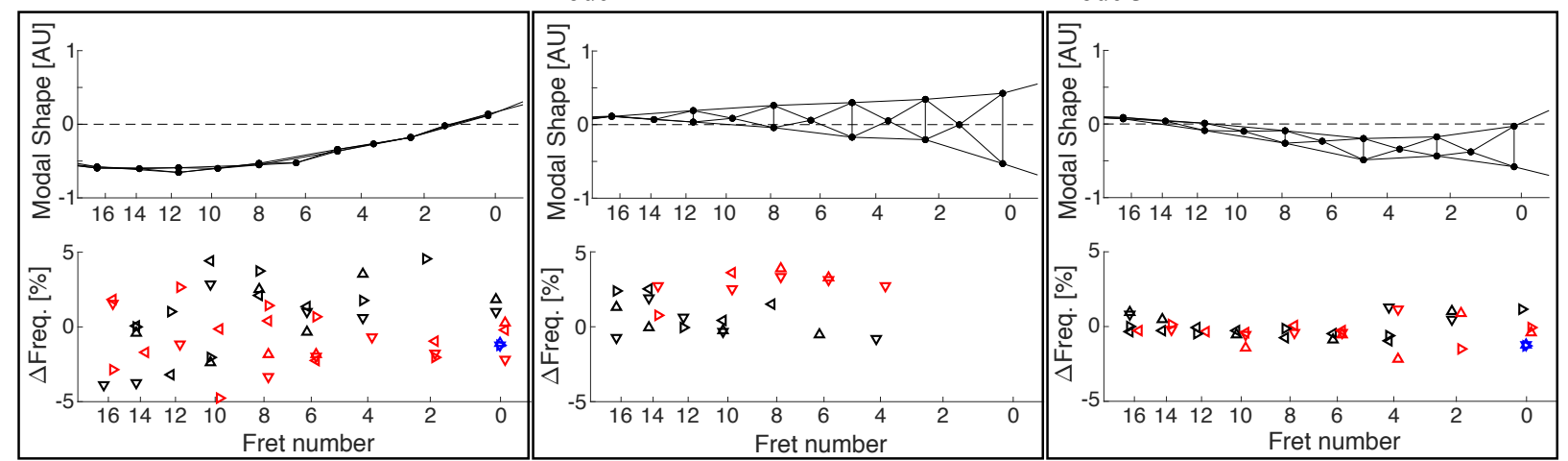

Mode 4

\section{Mode 5}

Mode 6
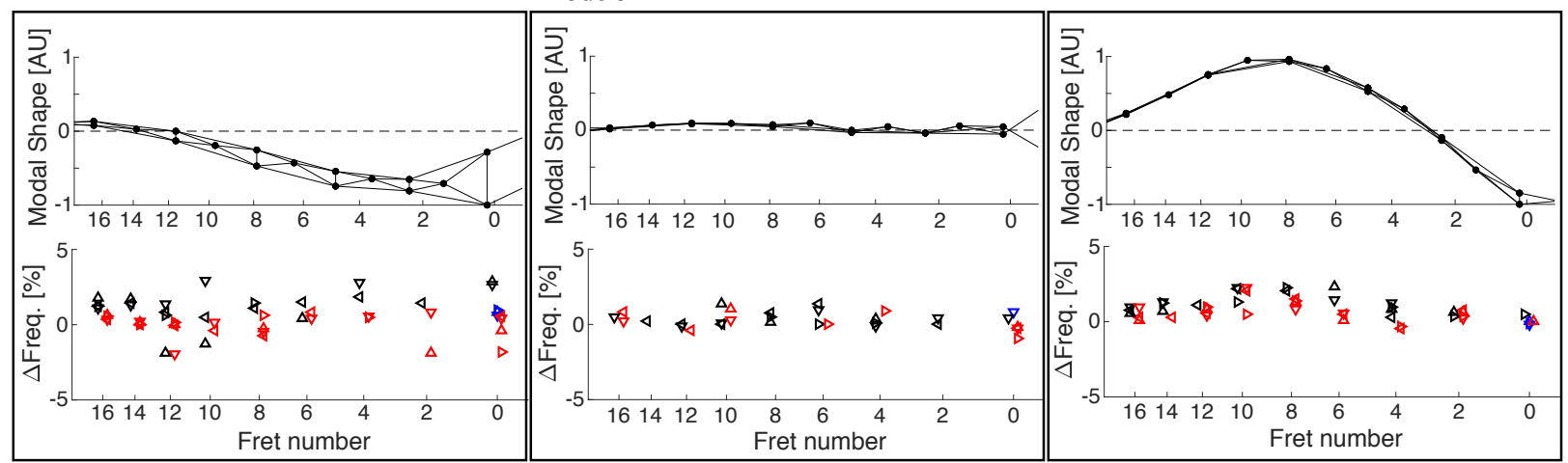

FIG. 5. [Color online] Frequency deviation identified by the OMA. For each mode, $\Delta$ Freq. is the frequency deviation, in percentage, from the modal frequency identified by the classical method (see Table 3). The results for standing player are plotted in black and for sitting player in red whereas the free-free configuration is plotted in blue. Different markers are used for the identification results on each plucked string: A2-string with $\triangleleft$, D3-string with $\nabla$, G3-string with $\triangle$, B3-string $\triangleright$. Dashed lines represent the mesh at rest position, black dots connected by solid lines represent the deformation corresponding to the modal shape on the $x z$-plane. 

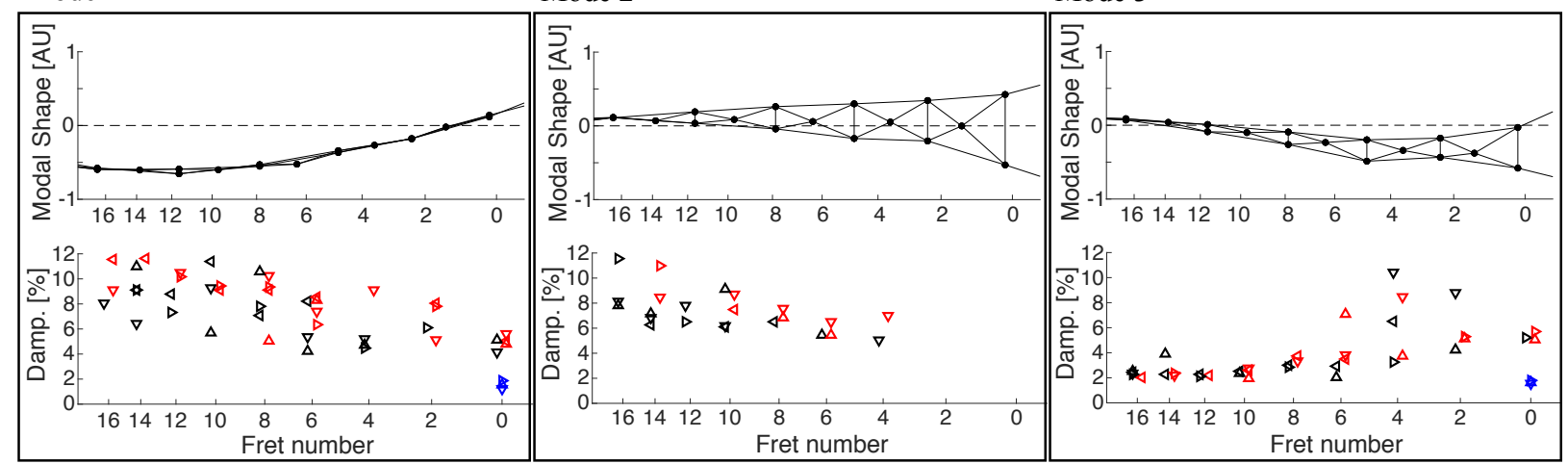

\section{Mode 4}

\section{Mode 5}

Mode 6
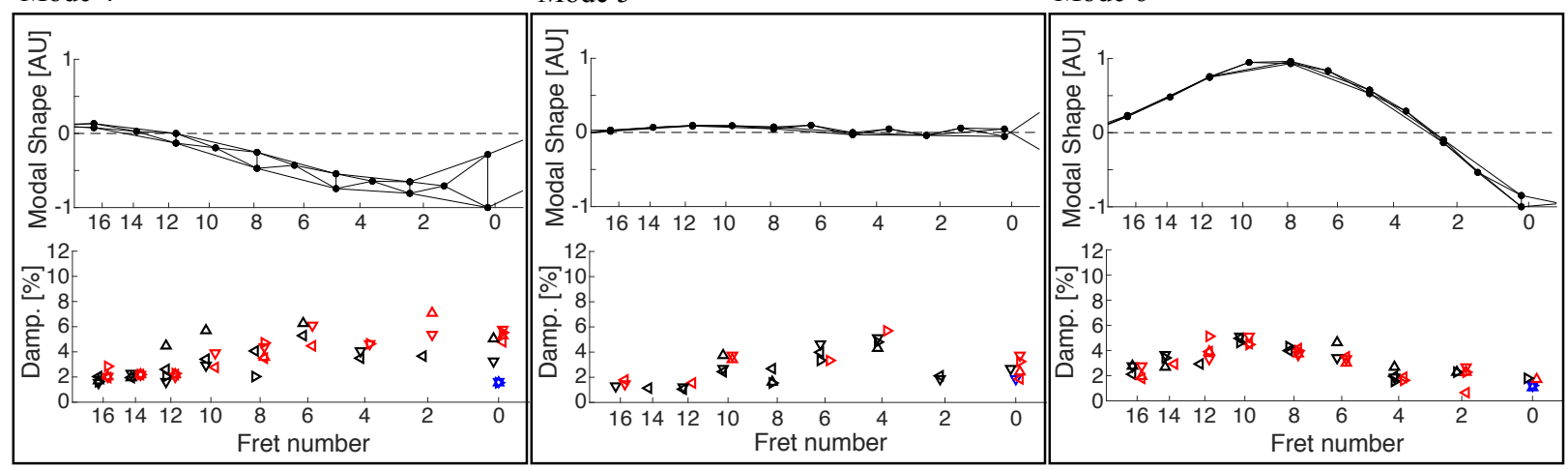

FIG. 6. [Color online] modal damping identified by the OMA. The results for standing player are plotted in black and for sitting player in red whereas the free-free configuration is plotted in blue.

Different markers are used for the identification results on each plucked string: A2-string with $\triangleleft$, D3-string with $\nabla$, G3-string with $\triangle$, B3-string $\triangleright$. Dashed lines represent the mesh at rest position, black dots connected by solid lines represent the deformation corresponding to the modal shape on the $x z$-plane.

\section{B. Operational Modal Analysis}

\section{Free-free configuration}

162 163

The OMA is applied on response signals measured on the electric guitar neck for four strings. In order to test the method, the OMA is first performed on an electric guitar on 
free-free conditions. For each of the four strings plucked, most of the modes are identified. Figure 5 and Figure 6 show modal frequency deviation and modal damping, with a marker for each string, and in blue for the free-free measurement at the nut (fret 0). Blue markers are generally superimposed, showing that OMA for structural modes of the instrument do not depend strongly on which string is used for the excitation and, therefore, a perfect reproducibility of the method. Moreover, modal frequency and damping are found to be very close to those of the classical modal analysis in comparison to previous results obtained for a concert harp (Chomette and Le Carrou, 2015). Note that for torsional modes, no physical poles are found for mode 2 and only one with D3-string for mode 5 by the OMA algorithm. These modes seem to be not well excited by the strings contrary to bending modes.

\section{Playing configuration}

The OMA is then applied on accelerometer measurements when the electric guitar is played by a person standing or sitting. Modal frequency deviation and damping for all left-hand positions along the neck and for all player configurations are gathered in Figures 5 and 6 for the first six modes.On these figures, the color of the maker defines the players configuration, "standing" in black and "sitting" in red, and the markers' shape indicates which string was played for the OMA identification. In order to facilitate the interpretation of the modal frequency deviation and damping, the modal shape of each mode is also shown according to the neck cross-section on $x z$-plane (see Figure 1) between the 16th fret and the nut. 
Mode 4
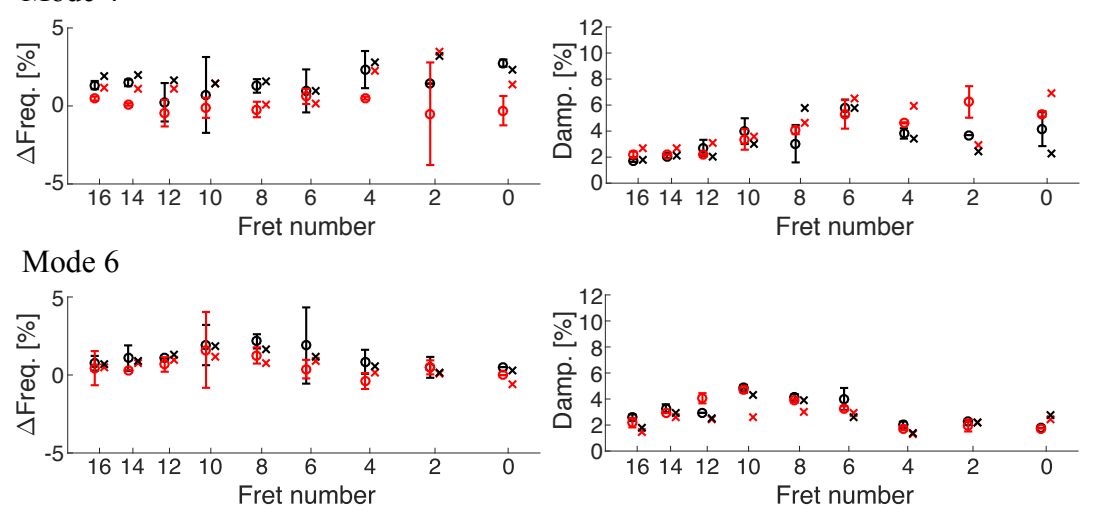

FIG. 7. [Color online] Comparison between modal frequency deviation ( $\Delta$ Freq.) and modal damping identified by the OMA (circles) and by the classical modal analysis (crosses) for standing (black) and sitting (red) player. For OMA, the circles are the mean value of 4 measurements (on 4 strings) and the error bars show the expanded uncertainty with $95 \%$ confidence.

All modes are well identified by OMA with each of the four strings as an excitation. Some modes are however better identified than others, and this is due to a) the string/neck coupling point location with respect to the modal shape, b) the fact that torsional modes are generally excited less than bending modes. Modal frequencies are then found close to modal frequency in free-free condition by OMA or classical modal analysis with a variation less than $5 \%$ for modes 1 and 2 and less than $2.5 \%$ for modes 3 to 6 . This small impact of the player on the modal frequencies has already been noted for local modes on an electric bass (Fleischer, 2005). The variation range across frets in modal frequency and damping i) depends on the mode, and ii) is much higher than for free-free conditions. 


\section{DISCUSSION}

But more generally, the variation of modal damping with the note played (pressed fret) seems to exhibit a systematic behavior. This evolution is found to be directly linked to the modal shape: the wider the mode shape displacement is at fingering location, the higher the damping is. When the player's left hand is close to a node of the mode shape, the modal damping is little affected as shown for mode 3 , frets 10 to 16 , or for mode 4 , fret 14 or mode 6 frets 2 and 4 . Neck modal dampings are modified by the hand grasping the neck and the finger pressing the fingerboard, only if the modal displacement is large enough at the fretting point. For mode 1, the guitar's body has a significant displacement. Therefore, for this mode, the stomach touching the body acts as an additional dashpot. That might be why, for mode 1 , the modal damping in playing configuration is found to always be higher than the modal damping without player (classical modal analysis) at about $5 \%$ for fret 0 (close to the modal node). Note that for a sitting configuration, the thigh also touches the guitar's body and increases the damping for this mode. Given the increased number of player/instrument contact points, this player configuration does not generally imply a higher damping than when the player is standing, as these contact points (see Figure 2-(d)) are close to a nodal line of mode 2 (see Figure 3).

Concerning the additional stiffness brought by the left-hand and the strap, a detailed analysis of the modal frequencies identified by OMA can provide answers. Indeed, at fret 0 , the player does not press the string on the fingerboard. Therefore, the increase in modal frequency is only due to the constraint applied by the strap on the guitar body. For the 
other frets, when the fingers act on the neck close to a node, modal frequency is found to be higher than for the sitting player (see, for instance, Fret 2 of Mode 1 or Fret 14 and 16 for mode 4), confirming that the strap brings an additional stiffness at the body in playing configuration. When the finger presses on the fretboard close to an anti-node, no systematic and significant modal frequency evolution is found with respect to the free-free configuration for modes $1,3,4$, and 6 . Torsional mode 2 shows a noteworthy separation between modal frequencies for each playing configuration. Modal frequencies are higher when the player is sitting than when standing. This could be due to changes in grip force between these two configurations. Grip force is presumably higher when the strap does not hold the guitar (sitting configuration, compared to standing configuration with strap). We previously encountered the same modal frequency evolution for torsional modes of more or less strongly held tennis rackets (Chadefaux et al., 2017). This interpretation is consistent with what the player felt during the experiment. For this particular mode, the left-hand seems to bring some additional stiffness at the neck.

In this paper, two methods are used to identify the modal parameters of the electric guitar when playing: in real and in mimic situations when the player is sitting or standing. In order to compare them, modal parameters for modes 4 and 6 are plotted in Figure 7. OMA results are gathered, for each fret, as the mean value of the frequency and of the damping computed from those identified from the 4 strings plucked, as shown in Figures 5 and 6. In Figure 7, error bars indicate the dispersion of the results and show, to some extent, that the dynamic behavior of the instrument also exhibits a variability that depends on all the contact points between the instrument and the player (hand-instrument neck, stomach-instrument body, 
thigh-instrument body). On the whole, the results obtained by the two methods are found to be very close. In order to have a global estimation of the influence of the player on the modal parameters of the instrument, the mimic situation with a classical modal analysis can be a good approximation.

Getting back to sound, we recall that if the electric guitar is heard through a loudspeaker, the sound originates in the mechanical vibration of the string (sensed by the magnetic pickup). The string is attached to the instrument at both ends. In other words string and structure are coupled and the string's modal parameters are modified by the presence of the structure (Fleischer and Zwicker, 1998, 1999; Paté et al., 2014). Former studies showed that the string's modal dampings (much more than the frequencies) depend on the conductance (real part of the admittance) measured at the string/structure contact point on the neck (much more than on the bridge). When some string and structure frequencies come close to one another, the corresponding string partial gets abnormally damped (Paté et al., 2014). This results in timbre changes (if string partials are damped) or decay times strong reduction (if fundamental frequency is damped). The latter phenomenon is also called "dead spot", and should be avoided (by e.g. detuning the string or modifying the structure in order to push the frequencies further apart). (Fleischer and Zwicker, 1998, 1999) characterized dead spots with the "T30" (time needed by the signal to decrease by 30dB from its maximum level). In order to predict dead spot occurrences, (Paté et al., 2014) proposed a sound synthesis model for the computation of the T30 that we reuse here. 
The synthetic string signal is computed for G3 string from fret 0 (nut) to fret 16 every two frets (in order to correspond to the points of vibratory measurement) as a sum of quasi-harmonic damped sinusoids:

$$
s(t)=\sum \frac{\sin \left(2 \pi f_{n} t\right)}{n} e^{-2 \pi f_{n} \xi_{n} t}
$$

where (see Equation 22 in (Paté et al., 2014)):

- the amplitude of the fundamental component is set to 1 and the amplitude of rank- $n$ partial is $\frac{1}{n}$;

- string modal dampings $\xi_{n}$ are the sum of isolated string dampings $\xi_{0, n}$ (measured in (Paté et al., 2014), e.g. Figure 5) and additional damping due to the structure $\xi_{\text {struct }, n}=\operatorname{Re}[Y] \frac{c^{2} \rho_{L}}{2 \pi L f_{n}}$, where $\mathrm{Y}$ is the driving-point admittance (defined below), where $c$ is the wave velocity in the string, $L$ is the vibrating length (changing for each fret), $\rho_{L}$ the string's mass per unit length (see Equation 20 in (Paté et al., 2014));

- the frequency of rank- $n$ partial $f_{n}$ equals $\frac{n c}{2 L}\left[1+\frac{n^{2} \pi^{2} E I}{2 L^{2} T}+\frac{\sqrt{\rho_{L} T}}{n \pi} \operatorname{Im}[Y]\right]$, that is a stiff string model connected to a mechanical admittance, $E$ is the Young's modulus of the string's material, $I$ the string's second moment of area, $T$ the string tension (see Equation 19 (Paté et al., 2014));

- $\mathrm{Y}$ is the driving-point admittance at string/structure contact point, where both velocity and force are measured at the same point. In practice here, this quantity is synthesized based on a modal fit of measurements done in free-free condition on the electric guitar structure in which modal dampings are replaced by the modal dampings measured in Subsection IV B 2 for sitting and standing musician; 
- the upper limit of the summation is $800 \mathrm{~Hz}$, which is close to the upper limit of the magnetic pickup (for the present pickup $1000 \mathrm{~Hz}$, see (Paté et al., 2014)), and which roughly corresponds to where modal overlap starts to hinder correct identification of modal parameters.

For each of these 9 synthetic signals ${ }^{1}$ (frets 0 to 16 by steps of 2), the energy decay curve (EDC) is computed using the backwards integration method (Schroeder, 1965), then the T30s are computed from a linear regression on the EDCs. T30 values are shown in Figure 8. T30 ranges from 3s to 8s for free-free configuration, which is in agreement with results obtained for a similar guitar in (Paté et al., 2014). A deadspot appears at fret 12 (lower T30 value), which is due to a coupling between structural mode 6 at around $373.81 \mathrm{~Hz}$ and fundamental frequency of note G4 at $392.00 \mathrm{~Hz}$ (see Table I). In general, T30 for sitting and standing musician is higher than for free-free configuration. This shows that the sound of the electric guitar may depend on the presence of a musician. However, differences between musicians' standing and sitting positions are very small, suggesting that the position of the musician has very little influence on the sound. When the electric guitar is held by a musician, the structure is damped and the conductance magnitude is lowered, reducing the influence of the coupling: different positions might well reduce the coupling by the same amount.

\section{CONCLUSION}

In this article, we presented an original work studying the influence of the guitarist on the dynamic behavior of the electric guitar structure and, by extension, on the sound of 


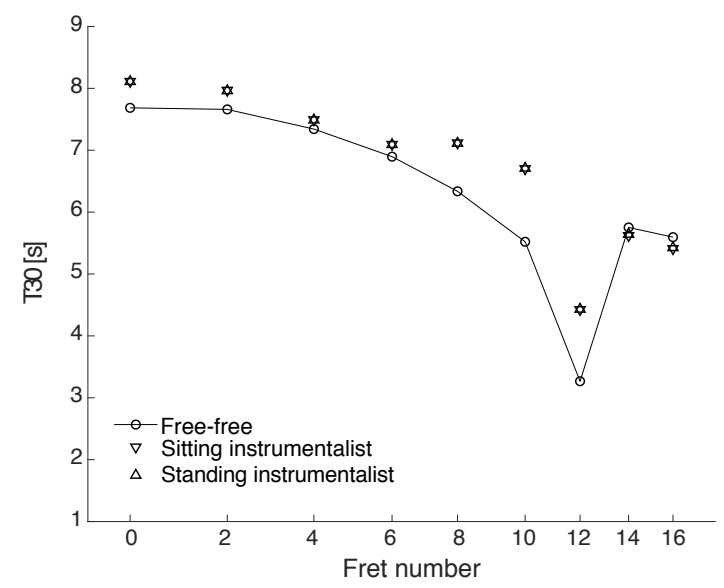

FIG. 8. T30 for each measured fret along string G3. Circles, downwards and upwards pointing triangles indicate T30 values computed from the synthesized signal for the electric guitar in free-free condition, held by a sitting musician, held by a standing musician respectively.

the instrument. Modal parameters, frequency and damping, were derived from accelerometer measurements of the structure when the player plays or simulates to play in different configurations.

As expected, the player damps the structure. But, in details, with his or her left hand, this additional damping evolves differently along the neck (i.e. at different positions on the modal shape). When the player is standing, the strap, holding the electric guitar, applies a constraint that brings an additional stiffness, effective for particular modes, to the structure. These electric guitar dynamic modifications may have some consequences for the sound of the instrument. By using a previously developed model, the decay time of the sound, which is a relevant sound indicator for the electric guitar, is higher in playing configuration than in free-free configuration, but independent of the guitarist's position. All these results were obtained by using a specific modal analysis method that is accurate and 
usable in playing configuration. Although less accurate, a classical modal analysis could be used with a player simulating a playing configuration, in order to estimate with a quite great precision the player's influence on the dynamic behavior of the musical instrument. The OMA approach, for its part, brings subtle variations associated with player-structure interaction, for particular mode and fret combinations.

The influence of the player can now be integrated in physically-based sound synthesis algorithms or directly in instruments using active modal control by modifying modal damping or/and modal stiffness. The method developed here can be generalized to other musical instruments, like e.g. the classical guitar or instruments of the string quartet, or other kinds of structures handled by humans such as sport equipments where it is essential to have a knowledge of the dynamic behavior of the object (tennis racket, baseball bat, etc.) when it is held thus modified by the user, so as to quantify the vibration to which the user is exposed.

\section{ACKNOWLEDGMENTS}

The authors kindly thank the luthier Florent Guesdon who made and lent the guitar for the present study, as well as his instructors Yann-David Esmans and Fred Pons at Itemm. 


\section{REFERENCES}

${ }^{1}$ Note that computation of all $\mathrm{f}_{n}$ 's of these synthetic signals in the frequency range of the study showed that ratios between coupled string frequencies and corresponding isolated string frequencies were consistently less than 1.2 cent, which can not be perceived.

Benacchio, S., Mamou-Mani, A., Chomette, B., and Finel, V. (2016). "Active control and sound synthesis - two different ways to investigate the influence of the modal parameters of a guitar on its sound," Journal of the Acoustical Society of America 139(3), 1411-1419.

Brown, D., Allemang, R., Zimmerman, R., and Mergeay, M. (1979). "Parameter estimation techniques for modal analysis," SAE Technical Paper 790221, 1-19.

Chadefaux, D., Rao, G., Carrou, J.-L. L., Berton, E., and Vigouroux, L. (2017). "The effects of player grip on the dynamic behaviour of a tennis racket," Journal of Sports Sciences 35(12), 1155-1164.

Chomette, B., and Le Carrou, J.-L. (2015). "Operational modal analysis applied to the concert harp," Mechanical Systems and Signal Processing 56-57, 81 - 91.

Chomette, B., and Mamou-Mani, A. (2018). "Modal control based on direct modal parameters estimation," Journal of Vibration and Control 24(12), 2389-2399.

Fleischer, H. (2005). "Vibration of an electric bass guitar," Acta Acustica United With Acustica 91(2), 247-260.

Fleischer, H., and Zwicker, T. (1998). "Mechanical vibrations of electric guitars," Acustica 84(4), 758-765. 
Fleischer, H., and Zwicker, T. (1999). "Investigating dead spots of electric guitars," Acustica 85(1), 128-135.

Gough, C. (1980). "Resonant response of a violin g-string and the excitation of the wolfnote," Acustica 44(2), 113-123.

Gough, C. (1981). "The theory of string resonancxes on musical-instruments," Acustica 49(2), 124-141.

Guillaume, P., Verboven, P., Vanlanduit, S., Van der Auweraer, H., and Peeters, B. (2003). "A poly-reference implementation of the least-squares complex frequency-domain estimator," in International Conference and Exposition on Structural Dynamics (IMAC), Society for Experimental Mechanics, Vol. 21, 183-192.

James, G., Carne, T., and Lauffer, J. (1995). "The Natural Excitation Technique (NExT) For Modal Parameter Extraction From Operating Structures," Modal Analysis-The International Journal Of Analytical And Experimental Modal Analysis 10(4), 260-277.

Marmaras, N., and Zarboutis, N. (1997). "Ergonomic redesign of the electric guitar," Applied Ergonomics 28(1), 59-67.

Marshall, H. (1986). "Operational modal analysis in the presence of harmonic excitation," Journal of the Catgut acoustical society 45, 28-33.

Mohanty, P., and Rixen, D. (2004). "Operational modal analysis in the presence of harmonic excitation," Journal of Sound and Vibration 270(1-2), 93-109.

Paté, A. (2014). "Lutherie de la guitare électrique solid body : aspects mécaniques et perceptifs [lutherie of the solid body electric guitar : mechanical and perceptual aspects]," Ph.D. thesis, univerisité Pierre et Marie Curie, Paris, France. 
370

371

372

Paté, A., Le Carrou, J.-L., and Fabre, B. (2014). "Predicting the decay time of solid body electric guitar tones," Journal Of The Acoustical Society Of America 135(5), 3045-3055.

Schroeder, M. R. (1965). "New method of measuring reverberation time," The Journal of the Acoustical Society of America 37(3), 409-412.

Woodhouse, J. (2004a). "On the synthesis of guitar plucks," Acta Acustica United With Acustica 90(5), 928-944.

Woodhouse, J. (2004b). "Plucked guitar transients: Comparison of measurements and synthesis," Acta Acustica United With Acustica 90(5), 945-965. 